\title{
171
}

\section{DOSAGGIO DEGLI ANTICORPI ANTI-CCP NELL'ARTRITE REUMATOIDE (AR) CONCLAMATA E ALL'ESORDIO: VALUTAZIONE E CONFRONTO CON METODI DIAGNOSTICI E STRUMENTALI.}

Biano A.'; Gera A. ${ }^{4}$ Scarso L. ${ }^{6}$; De Santis P.'; Delvino P.G. ${ }^{5}$; Filippini D. ${ }^{8}$;Venturino M. ${ }^{3}$; Garlaschi C. ${ }^{9}$; Pozzoli R. ${ }^{7}$

'Laboratorio di Patologia Clinica,

${ }^{2}$ Servizio di Day Hospital di Reumatologia,

3Unità di Diagnostica per Immagini

- Ospedale di Cairo Montenotte ASL 2 Savonese;

${ }^{4}$ Servizio di Microbiologia,

${ }^{5}$ Servizio di Reumatologia - ospedale S. Andrea ASL I I Vercelli;

${ }^{6}$ Centro di Immunoematologia e Trasfusionale- Istituto G.Gaslini Genova; "Servizio di Microbiologia e Virologia,

${ }^{8}$ Servizio di Reumatologia - Ospedale Riguarda Ca' Granda Milano; 'U.O. Microbiologia, A.O. ICP Milano

Obiettivi: valutazione delle caratteristiche di sensibilità, specificità degli anticorpi anti-CCP vs il Fattore Reumatoide (FR) IgM in soggetti affetti da Artrite Reumatoide (AR), Malattie Autoimmuni Sistemiche (MAIS) o donatori sani. Verifica dell'efficacia della determinazione degli anticorpi anti-CCP in esordio di malattia paragonata ad indagini di Imaging quali la Risonanza Magnetica. Definire un valore predittivo per il dosaggio degli anticorpi anti-CCP nei confronti delle forme erosive o non erosive.

Metodi: sono stati valutati 133 pazienti con AR, 67 con MAIS e 82 donatori sani per un totale di 282 soggetti ;su tutti sono stati eseguiti sia gli anticorpi anti-CCP che il FR; sono 
state incluse inoltre informazioni riguardanti la durata di malattia, la stadiazione radiologica e la presenza di erosioni articolari. Gli anticorpi anti CCP sono stati valutati con un kit ELISA (DiaSorin-Immunoscan RA, Eurodiagnostica) mentre il FR è stato dosato con tecnica turbidimetrica o nefelometrica.

Risultati: la sensibilità e la specificità degli anti-CCP nei soggetti con AR sono risultate rispettivamente $71.4 \%$ e $98.6 \%$ mentre per il FR sono risultate $72.9 \%$ e $72.8 \%$. I valori di predittività Positiva e Negativa sono risultati molto più alti per gli anticorpi anti CCP rispetto all'uso del solo FR (97.9\% e $79.2 \%$ vs. $70.8 \%$ e $74.8 \%$ ).

L'uso concomitante dei due marcatori diagnostici (anti CCP + FR) innalza la sensibilità diagnostica a $87.5 \%$ con un VPP di $86.6 \%$ e VPN di $99 \%$.

Lo studio della risonanza magnetica, sebbene ristretto a 5 casi in esordio di malattia, ha dimostrato una correlazione positiva tra fasi iniziali di AR (non ancora evidenziabili con radiologia tradizionale delle articolazioni metacarpofalangee) e la positività per anticorpi anti-CCP; è stato possibile correlare lo stadio funzionale di malattia (distinta tra forme erosive e non erosive)ai valori di anti-CCP.

Conclusioni: come già ampiamente descritto in letteratura il dosaggio degli anticorpi anti-CCP si dimostra un marcatore diagnostico molto utile, soprattutto in fase di esordio di malattia. 